\title{
Steatosis affects the performance of liver stiffness measurement for fibrosis assessment in patients with genotype 1 chronic hepatitis $\mathrm{C}$
}

\author{
Fabio Salvatore Macaluso ${ }^{1, *}$, Marcello Maida ${ }^{1}$, Calogero Cammà ${ }^{1}$, Giuseppe Cabibbo ${ }^{1}$, \\ Daniela Cabibi ${ }^{2}$, Rossella Alduino ${ }^{3}$, Vito Di Marco ${ }^{1}$, Antonio Craxì ${ }^{1}$, Salvatore Petta ${ }^{1}$ \\ ${ }^{1}$ Sezione di Gastroenterologia, Di.Bi.M.I.S., University of Palermo, Italy; ${ }^{2}$ Cattedra di Anatomia Patologica, University of Palermo, Italy; \\ ${ }^{3}$ Dipartimento di Scienze Economiche, Aziendali e Statistiche (SEAS), University of Palermo, Italy
}

Background \& Aims: In Chronic Hepatitis C (CHC), the influence of steatosis on liver stiffness measurement (LSM) is still debated. We assessed the impact of steatosis and its ultrasonographical sign - bright liver echo pattern (BLEP) - on LSM values and on transient elastography (TE) accuracy for the diagnosis of liver fibrosis, in a cohort of consecutive patients with Genotype 1 (G1) CHC.

Methods: Patients ( $n=618$ ) were assessed by clinical, ultrasonographic and histological (Scheuer score) features. TE was performed using the $\mathrm{M}$ probe.

Results: Male gender $(p=0.04)$, steatosis as continuous variable $(p<0.001)$, severity of necroinflammation $(p=0.02)$ and stage of fibrosis $(p<0.001)$ were associated with LSM by multivariate linear regression analysis. Among patients within the same fibrosis stages (F0-F2 and F3-F4; F0-F3 and F4), mean LSM values, expressed in $\mathrm{kPa}$, were significantly higher in subjects with moderate-severe steatosis ( $\geqslant 20 \%$ at liver biopsy) compared with those without, as well as in patients with BLEP on US compared with their counterpart. In subjects without severe fibrosis (F0F2) and without cirrhosis (F0-F3), a higher rate of false-positive LSM results was observed in patients with steatosis $\geqslant 20 \%$ compared with those without (F0-F2: $35.3 \%$ vs. 17.9\%; F0-F3: $38.9 \%$ vs. $16.6 \%$ ), and in patients with BLEP on US (F0-F2: $28.0 \%$ vs. 18.3\%; F0-F3: $29.7 \%$ vs. $17.8 \%$ ) compared with their counterpart. Conclusions: In patients with $\mathrm{G} 1 \mathrm{CHC}$, the presence of moderatesevere steatosis, detected by histology or by US, should always be taken into account in order to avoid overestimations of liver fibrosis assessed by TE.

(C) 2014 European Association for the Study of the Liver. Published by Elsevier B.V. All rights reserved.

Keywords: Evaluation; Non-invasive; Fibroscan ${ }^{\circledR}$; Ultrasound; Cirrhosis. Received 26 February 2014; received in revised form 14 April 2014; accepted 29 April 2014; available online 9 May 2014

* Corresponding author. Address: Sezione di Gastroenterologia, Di.Bi.M.I.S., Policlinico Universitario Paolo Giaccone, Piazza delle Cliniche, 2, 90127 Palermo, Italy. Tel.: +39091655 2274; fax: +390916552156.

E-mail address: fsmacaluso@gmail.com (F.S. Macaluso).

Abbreviations: AUROC, area under roc curve; BLEP, bright liver echo pattern; $\mathrm{CHC}$ chronic hepatitis C; G1, genotype 1; LR, likelihood ratio; LSM, liver stiffness measurement; TE, transient elastography; US, ultrasonography.

\section{Introduction}

The prognosis of patients with chronic liver diseases is critically decided by the amount of liver fibrosis that accumulates over the years as a consequence of several mechanisms of liver injury, with the ultimate occurrence of cirrhosis and its complications $[1,2]$. Liver biopsy is regarded as the gold standard procedure to assess presence and severity of all liver diseases, including chronic hepatitis $\mathrm{C}(\mathrm{CHC})$. However, it is an invasive procedure carrying a certain risk of complications, and its accuracy is affected by concerns related to inter- and/or intra-observer discrepancies [3], and by other factors such as length and width of biopsy sample, sampling errors, and inconsistency in defining histological features due to the variety of the several available scoring systems $[4,5]$. Furthermore, new therapeutic strategies against HCV infection at high efficacy and with an excellent profile of tolerability will be available in the near future; consequently, the need for histological staging will decrease, and non-invasive techniques for evaluation and monitoring of patients with CHC, particularly transient elastography (TE) [6] and ultrasonography (US) [7], will play an increasingly relevant role.

The use of TE to estimate liver fibrosis has been repeatedly validated in different settings [8], including CHC [9-11]. Independently of the underlying etiology, TE showed overall a good accuracy in diagnosing severe fibrosis and cirrhosis [8,12,13], even if its performance seems to be affected by several factors, such as alanine aminotransferase flares and severe liver necroinflammation [14-16], recent food intake [17], hepatic congestion [18], extrahepatic cholestasis [19], high body mass index (BMI) $[20,21]$ and insulin resistance [22]. Conversely, there are controversial data about the influence of liver steatosis on LSM values and TE performance, especially in patients with $\mathrm{CHC}$. Indeed, even if a lower interobserver agreement for LSM was observed in patients with liver steatosis compared with their counterpart [23], several studies showed no impact of steatosis on liver stiffness $[10,24]$, others demonstrated increased LSM values in presence of high degrees of steatosis [25-27], whereas steatosis $>33 \%$ was associated with lower LSM values in NAFLD patients with severe fibrosis [28].

The aim of the present study was to assess the impact of liver steatosis, detected by histology and US, on LSM values and on 


\section{Research Article}

accuracy of TE for fibrosis diagnosis in a cohort of consecutive biopsy-proven patients with genotype 1 (G1) CHC.

\section{Patients and methods}

Patients

The study assessed consecutive patients with biopsy-proven G1 CHC, all recruited at the Gastrointestinal \& Liver Unit at the University Hospital in Palermo, and fulfilling all inclusion and exclusion criteria detailed below. Patients were included if they had a histological diagnosis of CHC (any degree of fibrosis, including cirrhosis) on a liver biopsy. G1 CHC patients were defined by the presence of serum anti-HCV and HCV-RNA, with persistently abnormal alanine aminotransferase (ALT), and by alcohol consumption of $<20 \mathrm{~g} /$ day in the last year or more, evaluated by a specific questionnaire. Exclusion criteria were: (I) advanced cirrhosis (Child-Pugh B and C); (II) hepatocellular carcinoma; (III) other causes of liver disease, mixed etiologies and acute liver injuries of any etiology on chronic liver damage, (IV) human immunodeficiency virus infection; (V) previous and/or current use of steatosis-inducing drugs or potentially hepatotoxic drugs, evaluated by interview; (VI) active intravenous drug addiction.

The study was performed in accordance with the principles of the Helsinki Declaration and its appendices, and with local and national laws, and written informed consent was obtained from all patients.

\section{Clinical and laboratory assessment}

Demographic, clinical and anthropometric data were collected at the time of liver biopsy. The diagnosis of arterial hypertension was based on a systolic blood pressure $\geqslant 135 \mathrm{mmHg}$ and/or diastolic blood pressure $\geqslant 85 \mathrm{mmHg}$ (measured three times within 30 min using a brachial sphygmomanometer), or on use of bloodpressure-lowering agents. The diagnosis of type 2 diabetes was based on the revised criteria of the American Diabetes Association, using a value of fasting blood glucose $\geqslant 126 \mathrm{mg} / \mathrm{dl}$ on at least two occasions [29]. A current therapy with insulin or oral hypoglycemic agents was documented in patients with a previous diagnosis of type 2 diabetes.

A 12-h overnight fasting blood sample was drawn at the time of biopsy to determine serum levels of ALT, platelet count, total cholesterol, HDL- and LDLcholesterol, triglycerides, blood glucose and insulin. IR was assessed with the homeostasis model assessment (HOMA) method through the following equation [30]: Insulin Resistance $($ HOMA-IR) $=$ Fasting insulin $(\mu \mathrm{U} / \mathrm{ml}) \times$ Fasting glucose $(\mathrm{mmol} / \mathrm{L}) / 22.5$.

All patients were tested at the time of biopsy for HCV-RNA (RT-PCR homemade; limit of detection: $12 \mathrm{IU} / \mathrm{ml}$ ). Genotyping was performed by INNO-LiPA, HCV II, Bayer.

Liver stiffness measurement

Transient elastography was performed with the Fibroscan ${ }^{\circledR}$ (Echosens, Paris, France) medical device, using the $M$ probe (also named as standard probe) LSM was assessed on the same day of liver biopsy, before the procedure and after an overnight fast, by trained operators who had previously performed at least 300 determinations in patients with chronic liver disease. As suggested by the manufacturing company and as reported in the literature [9], we considered 10 successful acquisitions with a success rate of at least $60 \%$, and with an interquartile range lower than $30 \%$, as representative measurements.

\section{Ultrasound assessment}

Ultrasound assessment was performed in the morning, on the same day of liver biopsy, by one operator (G.C.) trained for ultrasound techniques and particularly dedicated to liver examination, using a real-time Hitachi H21 apparatus with a $2-$ $5 \mathrm{MHz}$, convex, multi-frequency probe. Presence of hepatic steatosis was defined through detection of Bright Liver Echo Pattern (BLEP), i.e., fine, packed and high amplitude echoes, with consequent brightness of liver, increase in liver-kidney contrast and possible evidence of vascular blurring and deep attenuation signs [31].

\section{Histology assessment}

All slides were evaluated by an expert pathologist (D.C.), blinded to identity, US features and LSM values of the patients. Biopsies were classified according to Scheuer numerical scoring system [32]. Steatosis was defined as the percentage of hepatocytes containing fat droplets, and assessed as continuous variable. It was also classified as present at $\geqslant 5 \%$, significative at $\geqslant 10 \%$, moderate-severe at $\geqslant 20 \%$, and severe at $\geqslant 30 \%$.

Statistics

Continuous variables were summarized as mean \pm standard deviation and categorical variables as frequency and percentage. The $t$ test and $\chi^{2}$ test were used as appropriate. Multiple logistic regression models were used to assess the variables independently associated with different stages of fibrosis. In these models, the dependent variables were significant fibrosis, coded as $0=F 0$ to F1 and $1=F 2$ to F4; severe fibrosis, coded as $0=\mathrm{F} 0$ to F2 and $1=\mathrm{F} 3$ to F4; and cirrhosis, coded as $0=\mathrm{F} 0$ to $\mathrm{F} 3$ and $1=\mathrm{F} 4$. As candidate risk factors, we selected age, gender, BMI, baseline ALT, platelet count levels, total, LDL-and HDL-cholesterol, triglycerides, blood glucose, insulin, HOMA score, arterial hypertension, diabetes, HCV-RNA (expressed as Log10), severe grading and moderate-severe steatosis. In addition, a multiple linear regression analysis was performed to identify independent predictors of LSM as continuous dependent variable. As candidate risk factors, we selected the same variables included in the above models and added stage of fibrosis as an additional independent variable. To avoid the effect of collinearity, HOMA score, blood glucose levels and insulin levels were not included in the same multivariate model. Receiver operating characteristic (ROC) curves were applied to find the best cut-off values and to identify the area under ROC curve (AUROC) of LSM able to discriminate the different stages of fibrosis. Furthermore, descriptions of the operating characteristics (sensitivity, specificity, positive predictive value [PPV], negative predictive value [NPV], positive likelihood ratio [LR+] and negative likelihood ratio [LR-]) of US for the detection of steatosis $\geqslant 5 \%$, $\geqslant 10 \%, \geqslant 20 \%$, and $\geqslant 30 \%$ were calculated, with the assumption that gold standard for the diagnosis of steatosis was histologic examination. Finally, multiple logistic regression models for LSM values were performed, using the best LSM cut-offs for discriminating severe fibrosis and cirrhosis as dependent variables. All analysis were performed using SPSS v. 20.0 statistical package for Macintosh (SPSS Inc., Chicago, USA).

\section{Results}

Patients

From January 2008 to October 2013, we included 702 consecutive patients with G1 CHC who underwent TE, US, and liver biopsy. Eighty-four (12\%) failed to obtain 10 valid LSM acquisitions due to obesity or to unreliable results according to manufacturer's recommendations (see above). Hence, 618 patients with valid LSM acquisitions could be included in the analysis. Table 1 summarizes the baseline features of these 618 patients. Mean age was $53.0 \pm 12.1$ years, with a male to female ratio approximately equal. More than half of patients was in the overweight or obese range, a quarter was hypertensive and diabetes was present in about thirteen percent of subjects. Mean values for total, HDLand LDL-cholesterol, and triglycerides were within the normal range, whereas mean HOMA values were elevated $(3.2 \pm 2.5)$.

At liver biopsy, approximately one patient in four had fibrosis $\geqslant 3$ by Scheuer score, whereas prevalence of severe necroinflammation (grading 3 ) was of $33.8 \%$. About half of the cases had histological evidence of steatosis, which was of moderate-severe grade $(\geqslant 20 \%)$ in 155 cases $(25.1 \%)$.

\section{Factors associated with fibrosis}

Univariate and multivariate regression analyses were performed to identify potential associations between each feature of patients 
Table 1. Baseline features of 618 patients with genotype 1 chronic hepatitis C.

\begin{tabular}{|c|c|}
\hline Variable & $\begin{array}{l}\text { Genotype } 1 \\
\text { chronic hepatitis C } \\
(n=618)\end{array}$ \\
\hline Age - yr & $53.0 \pm 12.1$ \\
\hline Male sex & $330(53.4 \%)$ \\
\hline Body Mass Index - kg/m² & $25.8 \pm 3.7$ \\
\hline $\begin{array}{l}\text { Body Mass Index }-\mathrm{kg} / \mathrm{m}^{2} \\
\quad<25 \\
\quad 25-29.9 \\
\quad \geq 30\end{array}$ & $\begin{array}{l}266(43.1 \%) \\
269(43.5 \%) \\
83(13.4 \%)\end{array}$ \\
\hline Arterial hypertension & $163(25.4 \%)$ \\
\hline Type 2 diabetes & $79(12.8 \%)$ \\
\hline Alanine aminotransferase - IU/L & $90.9 \pm 82.7$ \\
\hline Platelet count $-\times 10^{3} / \mathrm{mm}^{3}$ & $203.9 \pm 117.1$ \\
\hline Cholesterol - mg/dl & $174.8 \pm 35.8$ \\
\hline HDL cholesterol - mg/dl & $55.0 \pm 17.6$ \\
\hline LDL cholesterol - mg/dl & $100.5 \pm 33.1$ \\
\hline Triglycerides - mg/dl & $96.2 \pm 48.0$ \\
\hline Blood glucose - mg/dl & $96.9 \pm 31.8$ \\
\hline Insulin $-\mu U / m l$ & $13.1 \pm 8.3$ \\
\hline HOMA & $3.2 \pm 2.5$ \\
\hline $\log _{10} \mathrm{HCV}-\mathrm{RNA}-\mathrm{IU} / \mathrm{ml}$ & $6.3 \pm 1.1$ \\
\hline Bright liver echo pattern on ultrasound & $206(33.3 \%)$ \\
\hline Liver stiffness - kPa & $9.3 \pm 5.3$ \\
\hline \multicolumn{2}{|l|}{ Histology at biopsy } \\
\hline \multicolumn{2}{|l|}{ Steatosis } \\
\hline Absent $(<5 \%)$ & $321(51.9 \%)$ \\
\hline Present $(\geq 5 \%)$ & $297(48.1 \%)$ \\
\hline Significative $(\geq 10 \%)$ & $263(42.6 \%)$ \\
\hline Moderate-severe $(\geq 20 \%)$ & $155(25.1 \%)$ \\
\hline Severe $(\geq 30 \%)$ & $95(15.4 \%)$ \\
\hline \multicolumn{2}{|l|}{ Grading } \\
\hline 1 & $115(18.6 \%)$ \\
\hline 2 & $294(47.6 \%)$ \\
\hline 3 & $209(33.8 \%)$ \\
\hline \multicolumn{2}{|l|}{ Stage of fibrosis } \\
\hline 0 & $39(6.3 \%)$ \\
\hline 1 & $167(27.0 \%)$ \\
\hline 2 & $237(38.3 \%)$ \\
\hline 3 & $110(17.8 \%)$ \\
\hline 4 & $65(10.5 \%)$ \\
\hline
\end{tabular}

yr, years; IU, international units; kPa, KiloPascal; HOMA, homeostasis model assessment; HDL, high density lipoprotein; LDL, low density lipoprotein. Data are given as mean \pm standard deviation or as number of cases (\%).

and liver fibrosis at three cut-off levels: (a) significant fibrosis (F2F4); (b) severe fibrosis (F3-F4) and (c) cirrhosis (F4) (Supplementary Table 1$)$. Liver stiffness remained significantly associated with significant (OR 1.188, 95\% CI 1.111-1.269; $p<0.001$ ) and severe (OR 1.330 , 95\% CI 1.248-1.416; $p<0.001$ ) fibrosis, and with cirrhosis (OR $1.235,95 \%$ CI 1.170-1.304; $p<0.001)$. The overall AUCs of these models were 0.780 (95\% CI $0.742-0.818$ ) for significant fibrosis, 0.891 (95\% CI 0.864-0.919) for severe fibrosis, and 0.898 (95\% CI 0.863-0.932) for cirrhosis.

Performance of US on steatosis assessment

BLEP was observed on US examination in 206 patients (33.3\%). Sensitivity, specificity, PPV, NPV, LR+ and LR- of US for the

\section{JOURNAL OF HEPATOLOGY}

detection of steatosis $\geqslant 5 \%, \geqslant 10 \%, \geqslant 20 \%$, and $\geqslant 30 \%$ are shown in Table 2. Notably, sensitivity, specificity and NPVs of US for the detection of both steatosis $\geqslant 20 \%$ and $\geqslant 30 \%$ were quite similar (67.7\%, $78.2 \%$, and $86.4 \%$ vs. $71.6 \%, 73.6 \%$, and $93.4 \%$, respectively).

Factors associated with LSM, and accuracy of TE for fibrosis assessment

Mean LSM was $9.3 \pm 5.3 \mathrm{kPa}$ (range 2.7-34.0). Liver stiffness values significantly increased according to fibrosis stage $(6.9 \pm 2.7$ in F1; $8.0 \pm 3.9$ in F2; $12.5 \pm 5.2$ in F3; $17.0 \pm 7.9$ in F4; $p<0.001$ ).

Older age, male gender, high BMI, high baseline ALT, low platelets, low cholesterol, high triglycerides, high blood glucose, high insulin, high HOMA, steatosis as continuous variable, severity of necroinflammatory activity, and stage of fibrosis were linked to LSM as continuous variable at univariate analysis $(p<0.10)$, even if only male gender $(p=0.04)$, steatosis as continuous variable $(p<0.001)$, severity of necroinflammation $(p=0.02)$, and stage of fibrosis $(p<0.001)$ remained associated by multivariate linear regression analysis.

ROC curves identified the best cut-offs of LSM able to maximize the accuracy of TE at $7.25 \mathrm{kPa}$ for the diagnosis of significant fibrosis (AUC 0.727 , sensitivity $66.3 \%$, specificity $66.5 \%$ ), at $8.95 \mathrm{kPa}$ for severe fibrosis (AUC 0.864 ; sensitivity $79.0 \%$, specificity $78.7 \%$ ) and at $10.65 \mathrm{kPa}$ (AUC 0.882 ; sensitivity $76.9 \%$, specificity 78.1\%) for cirrhosis. Considering the performance of LSM in discriminating significant fibrosis as not acceptable, we focused on severe fibrosis and cirrhosis only.

At the LSM cut-off of $8.95 \mathrm{kPa}$ for severe fibrosis, the falsepositive rate was $21.2 \%(94 / 443)$ and the false-negative rate was $20.6 \%$ (36/175). Concerning the performance of LSM $>10.65 \mathrm{kPa}$ for cirrhosis, the false-positive rate was $21.9 \%$ (121/ 553 ) and the false-negative rate was $23.1 \%$ (15/65). Fig. 1 summarizes the accuracy of LSM in diagnosing severe fibrosis (Fig. 1A) and cirrhosis (Fig. 1B) in the entire G1 CHC cohort. Of note, moderate-severe steatosis and severe grading, other than fibrosis stage, were significantly associated with LSM cut-offs of $>8.95 \mathrm{kPa}$ for severe fibrosis (OR 1.710; 95\% CI 1.026-2.655; $p=0.04$; and OR 2.696; 95\% CI 1.726-4.211; $p<0.001$, respectively - overall AUC of the model: 0.819 ) and of $>10.65 \mathrm{kPa}$ for cirrhosis (OR 2.119; 95\% CI 1.312-3.423; $p=0.002$; and OR 2.744; 95\% CI 1.769-4.255; $p<0.001$, respectively - overall AUC of the model: 0.827 ) by multivariate regression analyses.

Impact of liver steatosis and BLEP detection on LSM values and on accuracy of $T E$

Due to the independent association of liver stiffness with steatosis, we assessed the variations of LSM values and of TE accuracy according to presence or absence of moderate-severe steatosis $(\geqslant 20 \%)$ on histological examination, and according to BLEP detection on US, a reliable surrogate of steatosis.

Fig. 2 shows, in subgroups of patients with and without moderate-severe steatosis, mean LSM values among patients with F0F2 fibrosis stages ( $9.2 \pm 5.1$ vs. $7.0 \pm 2.8 ; p<0.001-$ Fig. $2 \mathrm{~A})$ and F3-F4 fibrosis stages (15.4 \pm 6.9 vs. $13.2 \pm 5.6 ; p=0.02 \quad-$ Fig. 2B), and among patients with F0-F3 fibrosis stages $(10.8 \pm 5.7$ vs. $7.7 \pm 3.5 ; p<0.001$ - Fig. 2C) and cirrhosis $(18.8 \pm 7.5$ vs. $15.9 \pm 6.1 ; p=0.09-$ Fig. 2D). As expected, similar results were obtained when the entire cohort was split according to presence or absence of BLEP on US (Fig. 3). Furthermore, 


\section{Research Article}

Table 2. Sensitivity, Specificity, Predictive Values and Likelihood Ratios of ultrasound for steatosis detection in genotype 1 chronic hepatitis $\mathrm{C}$ patients.

\begin{tabular}{lllllll}
\hline GI CHC $(n=618)$ & Sensitivity & Specificity & PPV & NPV & LR + & LR - \\
\hline Steatosis $\geq 5 \%$ & $172 / 297(57.9 \%)$ & $287 / 321(89.4 \%)$ & $172 / 206(83.5 \%)$ & $287 / 412(69.7 \%)$ & 5.46 & 0.47 \\
Steatosis $\geq 10 \%$ & $156 / 263(59.3 \%)$ & $305 / 355(85.9 \%)$ & $156 / 206(75.7 \%)$ & $305 / 412(74.0 \%)$ & 4.21 & 0.47 \\
Steatosis $\geq 20 \%$ & $105 / 155(67.7 \%)$ & $362 / 463(78.2 \%)$ & $105 / 206(51.0 \%)$ & $356 / 412(86.4 \%)$ & 3.11 & 0.41 \\
Steatosis $\geq 30 \%$ & $68 / 95(71.6 \%)$ & $385 / 523(73.6 \%)$ & $68 / 206(33.0 \%)$ & $385 / 412(93.4 \%)$ & 2.71 & 0.39 \\
\hline
\end{tabular}

PPV, Positive Predictive Value; NPV, Negative Predictive Value; LR+, Positive likelihood ratio; LR-, Negative likelihood ratio.
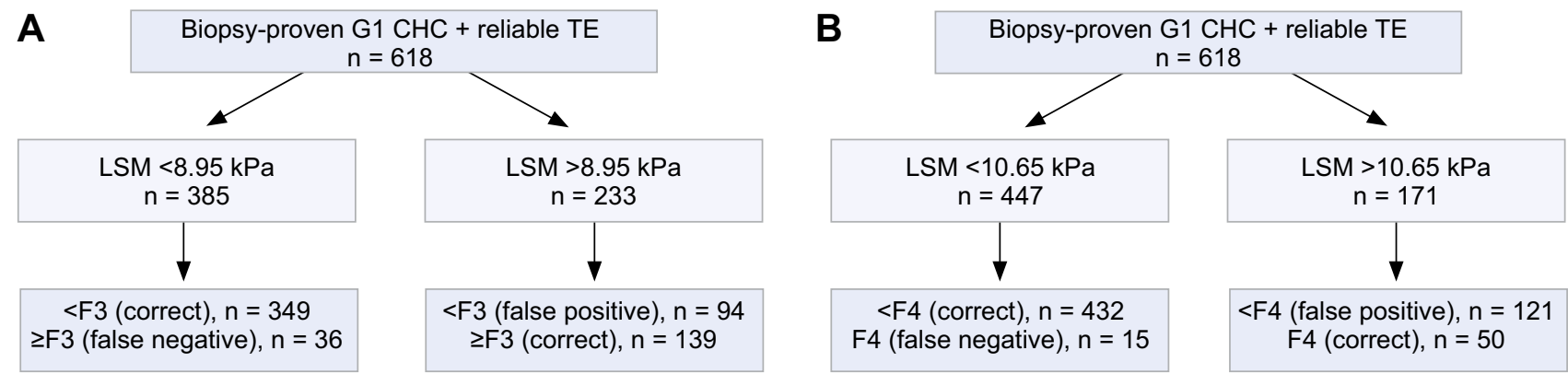

Fig. 1. Accuracy of transient elastography. For the prediction of severe fibrosis (A) and cirrhosis (B) in patients with genotype 1 chronic hepatitis $\mathrm{C}$ and reliable transient elastography.

among subjects without severe fibrosis (F0-F2), the rate of false positive LSM results was higher in patients with steatosis $\geqslant 20 \%$ (30/85; 35.3\%) compared with their counterpart (64/357; $17.9 \%)$ (Fig. 4A), and in patients with detection of BLEP (37/132; $28.0 \%)$ compared with those without $(57 / 311 ; 18.3 \%$ ) (Fig. 4B). Similar differences in false positive rates were observed among patients without cirrhosis (F0-F3) (Fig. 4C and D). Conversely, the impact of moderate-severe steatosis and of BLEP detection on false negative LSM results was negligible (data not shown). In addition, ROC curves were applied to find the best cut-off values of LSM able to discriminate severe fibrosis and cirrhosis among subgroups of patients with and without detection of BLEP. In patients with detection of BLEP, using a LSM cut-off of $9.95 \mathrm{kPa}$ for severe fibrosis (AUC 0.840 , sensitivity $78.4 \%$, specificity $78.0 \%$ ) and of $15.35 \mathrm{kPa}$ for cirrhosis (AUC 0.880 , sensitivity $76.2 \%$, specificity $87.0 \%$, the false-positive rates decreased from $28.0 \%$ to $22.0 \%$, and from $29.7 \%$ to $13.0 \%$, respectively. In patients without BLEP, the false-positive rates increased from $18.3 \%$ to $21.5 \%$, and from $17.9 \%$ to $22.8 \%$ using a LSM cut-off of $8.75 \mathrm{kPa}$ for severe fibrosis (AUC 0.873 , sensitivity $83.3 \%$, specificity $78.4 \%$ ) and of $10.05 \mathrm{kPa}$ for cirrhosis (AUC 0.892 , sensitivity $81.8 \%$, specificity 78.0\%), respectively.

Finally, although male subjects showed slightly higher mean LSM values compared with females, a similar overall influence of steatosis and of BLEP detection on LSM values was observed when the entire cohort was stratified according to the gender (data not shown).

\section{Discussion}

In our cohort of 618 consecutive biopsy-proven patients with G1 CHC, we found that steatosis, diagnosed by histology or by US - detection of BLEP - is independently associated with increased LSM values. As a consequence, we reported higher rates of false positive LSM results for the non-invasive assessment of both severe fibrosis and cirrhosis by TE in patients with BLEP on US examination compared with their counterpart.

Transient elastography is a reliable tool to identify liver fibrosis and cirrhosis, especially in patients with $\mathrm{CHC}$, where it has been repeatedly validated [9-13]. In our cohort of G1 CHC patients, the accuracy of TE for the assessment of liver fibrosis was good in diagnosing severe fibrosis and cirrhosis, while it was quite low for significant fibrosis, as expressed by their AUROCs. These findings are in line with previously reported data from the literature [13]. Furthermore, we identified LSM values of $7.25 \mathrm{kPa}, 8.95 \mathrm{kPa}$, and $10.65 \mathrm{kPa}$ as the best cut-offs for discriminating significant fibrosis (F2-F4), severe fibrosis (F3-F4), and cirrhosis (F4), respectively. Once again, these LSM cut-off values were comparable with those identified in other cohorts [13]. Some variability may be ascribed to the different prevalence of significant fibrosis and of several factors influencing liver stiffness independently from the amount of liver fibrosis in the various examined cohorts.

An interesting finding emerging from our data is that the presence of liver steatosis not only is clearly independently associated with higher LSM, but it is even able to affect the overall diagnostic performance of TE in patients with G1 CHC, causing potential overestimations of liver fibrosis. In this line, literature data have already emphasized a significant reduction of TE reproducibility in patients with chronic liver diseases due to different etiologies and with steatosis [23]. In addition, higher LSM values in presence of liver steatosis have been already reported in some small $[25,27]$ and large [26] cohorts of CHC patients, even if they were not confirmed in other studies [10,24], whereas in NAFLD patients an inverse relation was highlighted [28]. Surely, the presence of fat droplets in the hepatocytes influences the architectural structure of the liver, thus potentially changing 
A

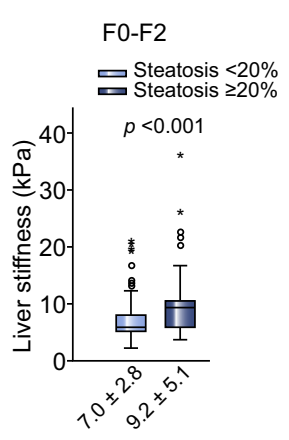

C

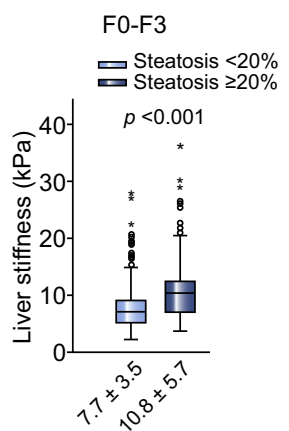

B

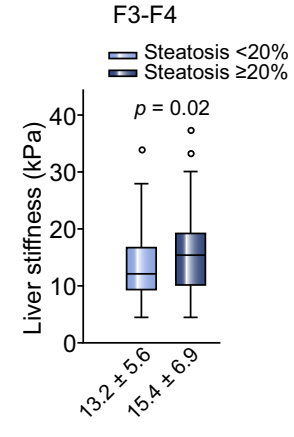

D

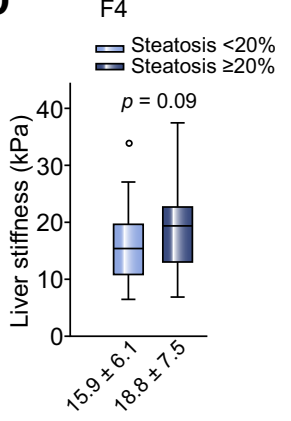

Fig. 2. Liver stiffness values distribution. Within patients with F0-F2 fibrosis stages (A) and F3-F4 fibrosis stages (B), and within patients with F0-F3 fibrosis stages (C) and cirrhosis (D), according to presence or absence of moderate-severe steatosis. The horizontal bar inside the box represents the mean value.

the propagation time of the vibratory wave through the liver, which is the key principle of TE. Nonetheless, the reason why this interference translated in a greater "softness", as observed in NAFLD patients [28], or conversely in a higher "hardness", as reported in our G1 CHC cohort, is not clear. Furthermore, we reported that the rates of false positive LSM results for the diagnosis of severe fibrosis and cirrhosis were strongly affected by the presence of moderate-severe steatosis. By contrast, a recent study on a multicenter large cohort of $\mathrm{CHC}$ patients [26] showed that the presence of significant steatosis induced false positive results in patients with F0-F1 fibrosis only, while it did not increase the rate of F0-F3 patients misclassified as cirrhotic. Differences in the prevalence of liver steatosis, and in the methods used to quantify both steatosis and fibrosis could explain the observed differences. In fact, Boursier and colleagues assessed all histological features by computerized morphometry, a technique reported as more sensitive than optical analysis [33], but not yet standardized and not used in clinical practice.

However, all the above quoted data raised the paradoxical problem that the performance of a non-invasive tool - TE - is affected by a variable - steatosis - arising from an invasive tool - liver biopsy. To overcome this issue, we tested the impact of a non-invasive evaluation of hepatic steatosis - US detection of BLEP - on TE performance. Accordingly, the most relevant finding arising from our analysis lies in the correlation of BLEP detection with both higher LSM values and false positive results. Specifically, when patients were divided according to presence or absence of BLEP on US, we found that the variations of LSM values and of TE accuracy were comparable with those observed when our cohort was splitted according to presence or absence of

\section{JOURNAL OF HEPATOLOGY}

A

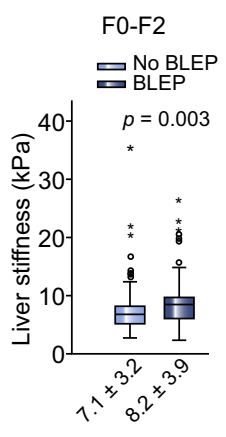

C

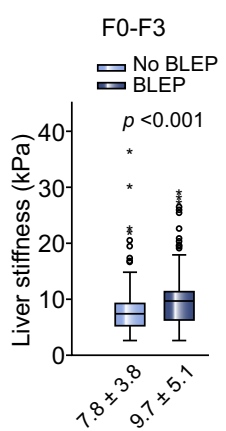

B

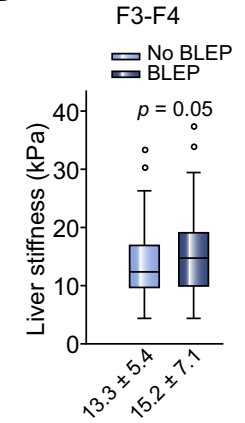

D

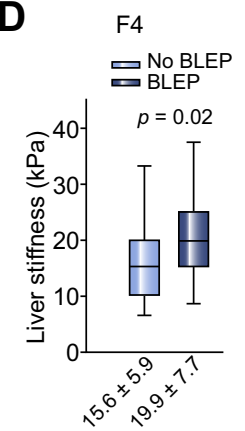

Fig. 3. Liver stiffness values distribution. Within patients with F0-F2 fibrosis stages (A) and F3-F4 fibrosis stages (B), and within patients with F0-F3 fibrosis stages (C) and cirrhosis (D), according to presence or absence of Bright Liver Echo Pattern (BLEP) on ultrasound. The horizontal bar inside the box represents the mean value.

moderate-severe steatosis, although at a slightly lesser extent. Provided that accuracy of US for the detection of steatosis is quite low in settings of CHC, especially due to poor PPVs for moderatesevere degrees of steatosis, as our group recently reported [7], we could speculate that BLEP may not be only the US expression of histological steatosis, but also of other known - liver necroinflammation, for example - or unknown factors able to increase LSM independently from the amount of fibrosis.

Consequently, from a clinical standpoint, our study suggests to interpret with caution TE findings in G1 CHC patients with detection of steatosis on US, in which LSM values could lead to an overestimation of liver fibrosis (Fig. 5). Interestingly, we obtained a clear decrease of false positive LSM results for the diagnosis of cirrhosis in patients with BLEP using a specific LSM cut-off value in this subgroup. Anyway, it should be acknowledged that the poor accuracy of US for the detection of steatosis in this setting represents a limitation when using US paired with LSM, since we risk losing a certain number of patients with steatosis and thus with potential overestimation of fibrosis assessed by LSM. Computed tomography and magnetic resonance imaging are able to demonstrate hepatic steatosis $[34,35]$ and may be useful in this setting, but their high costs and, for computed tomography, the risk of radiation exposure limit their applicability in routine clinical practice. CAP is likely a good alternative, although it was not evaluated in this study.

We should mention that there are some other limitations in our study. First, inter- and intra-observer agreement of LSM and US examinations were not assessed. Second, a comparison between histological/ultrasonographical findings and controlled 


\section{Research Article}

A
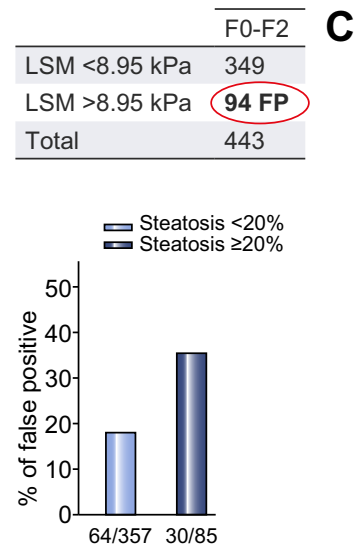

B

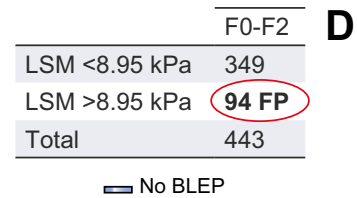

D

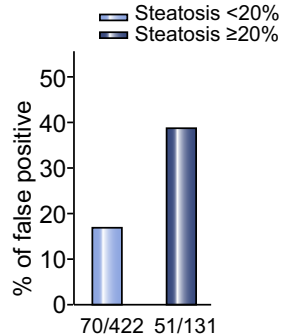

Another methodological issue could reside in the accuracy of liver biopsy examination related to sampling errors and intraobserver and inter-observer variability; in addition, the assessment of hepatic steatosis may be affected by the frequently uneven distribution of fat throughout the liver, and the quantification of hepatic steatosis as a percentage of steatotic hepatocytes may not represent a good marker of liver fat content because it does not take into account number and size of lipid droplets in single hepatocytes. A further methodological question is the potentially limited external validity of the results for different populations and settings. Our study included a cohort of Italian G1 CHC subjects, largely overweight, who were enrolled in a tertiary referral center for liver disease, limiting the broad application of the results. Finally, lack of follow-up data may limit the potency of our results.

In conclusion, although LSM is an useful and non-invasive tool for estimating the severity of $\mathrm{CHC}$, the presence of moderatesevere steatosis, per se or evaluated by US, should always be taken into account in order to avoid overestimations of liver fibrosis.

\section{Conflict of interest}

The authors who have taken part in this study declared that they do not have anything to disclose regarding funding or conflict of interest with respect to this manuscript.

\section{Supplementary data}

Fig. 4. Distribution of false-positive LSM results. In patients without severe fibrosis (F0-F2) according to presence or absence of moderate-severe steatosis (A) and to detection of Bright Liver Echo Pattern (BLEP) on ultrasound (B), and in patients without cirrhosis (F0-F3) according to presence or absence of moderatesevere steatosis (C) and to detection of Bright Liver Echo Pattern (BLEP) on ultrasound (D). (This figure appears in colour on the web.)

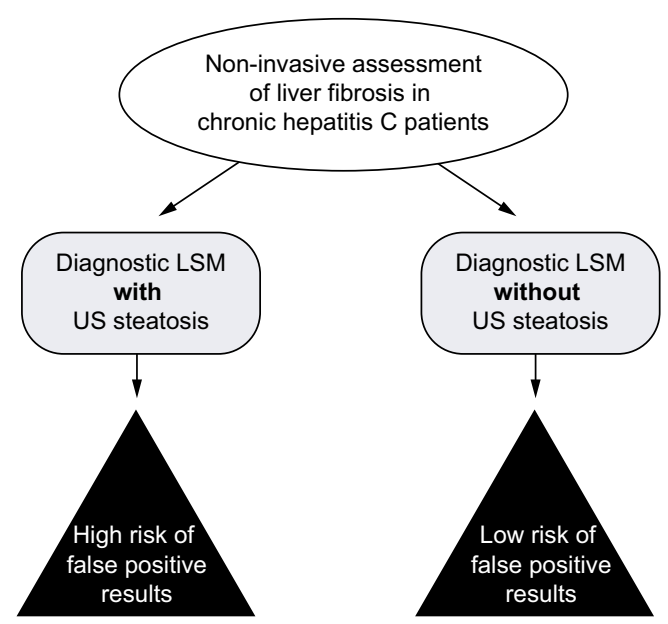

Fig. 5. Proposed diagnostic algorithm on the use of ultrasound for detection of steatosis paired with liver stiffness measurement.

attenuation parameter (CAP) data was not performed, as above mentioned, and the analysis of LSM was carried out using a conventional $\mathrm{M}$ probe only, while the XL probe was not used.
Supplementary data associated with this article can be found, in the online version, at http://dx.doi.org/10.1016/j.jhep.2014. 04.045 .

\section{References}

[1] Herzer K, Sprinzl MF, Galle PR. Hepatitis viruses: live and let die. Liver Int 2007;27:293-301.

[2] Petta S, Muratore C, Craxì A. Non-alcoholic fatty liver disease pathogenesis: the present and the future. Dig Liver Dis 2009;41:615-625.

[3] Manning DS, Afdhal NH. Diagnosis and quantitation of fibrosis. Gastroenterology 2008:134:1670-1681.

[4] Rousselet MC, Michalak S, Dupre F, Croué A, Bedossa P, Saint-André JP, et al. Hepatology 2005;41:257-264.

[5] Bedossa P, Dargère D, Paradis V. Sampling variability of liver fibrosis in chronic hepatitis C. Hepatology 2003;38:1449-1457.

[6] Sandrin L, Fourquet B, Hasquenoph JM, Yon S, Fournier C, Mal F, et al. Transient elastography: a new non-invasive method for assessment of hepatic fibrosis. Ultrasound Med Biol 2003;29:1705-1713.

[7] Macaluso FS, Maida M, Cammà C, Cabibi D, Alessi N, Cabibbo G, et al. Body mass index and liver stiffness affect accuracy of ultrasonography in detecting steatosis in patients with chronic HCV genotype 1 infection. Clin Gastroenterol Hepatol 2014;12:878-884.

[8] Talwalkar JA, Kurtz DM, Schoenleber SJ, West CP, Montori VM. Ultrasound based transient elastography for the detection of hepatic fibrosis: systematic review and meta-analysis. Clin Gastroenterol Hepatol 2007;5:1214-1220.

[9] Castéra L, Vergniol J, Foucher J, Le Bail B, Chanteloup E, Haaser M, et al. Prospective comparison of transient elastography, Fibrotest, APRI, and liver biopsy for the assessment of fibrosis in chronic hepatitis C. Gastroenterology 2005; $128: 343-350$.

[10] Arena U, Vizzutti F, Abraldes JG, Corti G, Stasi C, Moscarella S, et al. Reliability of transient elastography for the diagnosis of advanced fibrosis in chronic hepatitis C. Gut 2008;57:1288-1293. Sources of variability in histological scoring of chronic viral hepatitis. 


\section{JOURNAL OF HEPATOLOGY}

[11] Ziol M, Handra-Luca A, Kettaneh A, Christidis C, Mal F, Kazemi F, et al. Noninvasive assessment of liver fibrosis by measurement of stiffness in patients with chronic hepatitis C. Hepatology 2005;41:48-54.

[12] Friedrich-Rust M, Ong MF, Martens S, Sarrazin C, Bojunga J, Zeuzem S, et al. Performance of transient elastography for the staging of liver fibrosis: a meta-analysis. Gastroenterology 2008;134:960-974.

[13] Wong GL. Transient elastography: kill two birds with one stone? World J Hepatol 2013;5:264-274.

[14] Coco B, Oliveri F, Maina AM, Ciccorossi P, Sacco R, Colombatto P, et al Transient elastography: a new surrogate marker of liver fibrosis influenced by major changes of transaminases. J Viral Hepat 2007;14:360-369.

[15] Arena U, Vizzutti F, Corti G, Ambu S, Stasi C, Bresci S, et al. Acute viral hepatitis increases liver stiffness values measured by transient elastography. Hepatology 2008;47:380-384.

[16] Sagir A, Erhardt A, Schmitt M, Häussinger D. Transient elastography is unreliable for detection of cirrhosis in patients with acute liver damage. Hepatology 2008;47:592-595.

[17] Mederacke I, Wursthorn K, Kirschner J, Rifai K, Manns MP, Wedemeyer H, et al. Food intake increases liver stiffness in patients with chronic or resolved hepatitis C virus infection. Liver Int 2009;29:1500-1506.

[18] Lebray P, Varnous S, Charlotte F, Varaut A, Poynard T, Ratziu V. Liver stiffness is an unreliable marker of liver fibrosis in patients with cardiac insufficiency. Hepatology 2008;48:2089.

[19] Millonig G, Reimann FM, Friedrich S, Fonouni H, Mehrabi A, Büchler MW, et al. Extrahepatic cholestasis increases liver stiffness (Fibroscan ${ }^{\circledR}$ ) irrespective of fibrosis. Hepatology 2008;48:1718-1723.

[20] Wong GL, Chan HL, Choi PC, Chan AW, Lo AO, Chim AM, et al. Association between anthropometric parameters and measurements of liver stiffness by transient elastography. Clin Gastroenterol Hepatol 2013;11:295-302.

[21] Petta S, Di Marco V, Cammà C, Butera G, Cabibi D, Craxì A. Reliability of liver stiffness measurement in non-alcoholic fatty liver disease: the effects of body mass index. Aliment Pharmacol Ther 2011;33:1350-1360.

[22] Petta S, Cammà C, Di Marco V, Calvaruso V, Enea M, Bronte F, et al. Insulin resistance is a major determinant of liver stiffness in nondiabetic patients with HCV genotype 1 chronic hepatitis. Aliment Pharmacol Ther 2009;30:603-613.

[23] Fraquelli M, Rigamonti C, Casazza G, Conte D, Donato MF, Ronchi G, et al. Reproducibility of transient elastography in the evaluation of liver fibrosis in patients with chronic liver disease. Gut 2007;56:968-973.
[24] Wong VW, Vergniol J, Wong GL, Foucher J, Chan HL, Le Bail B, et al. Diagnosis of fibrosis and cirrhosis using liver stiffness measurement in nonalcoholic fatty liver disease. Hepatology 2010;51:454-462.

[25] Sanchez-Conde M, Montes Ramirez ML, Bellon Cano JM, Caminoa A Rodriguez FA, Garcia JC, et al. Impact of liver steatosis on the correlation between liver stiffness and fibrosis measured by transient elastography in patients coinfected with human immunodeficiency virus and hepatitis C virus. J Viral Hepat 2011;18:278-283.

[26] Boursier J, de Ledinghen V, Sturm N, Amrani L, Bacq Y, Sandrini J, et al Precise evaluation of liver histology by computerized morphometry shows that steatosis influences liver stiffness measured by transient elastography in chronic hepatitis C. J Gastroenterol 2014;49:527-537.

[27] Lupsor M, Badea R, Stefanescu H, Grigorescu M, Sparchez Z, Serban A, et al. Analysis of histopathological changes that influence liver stiffness in chronic hepatitis C Results from a cohort of 324 patients. J Gastrointestin Liver Dis 2008; 17:155-163.

[28] Gaia S, Carenzi S, Barilli AL, Bugianesi E, Smedile A, Brunello F, et al Reliability of transient elastography for the detection of fibrosis in nonalcoholic fatty liver disease and chronic viral hepatitis. J Hepatol 2011:54:64-71.

[29] American Diabetes Association. Report of the Expert Committee on the diagnosis and classification of diabetes mellitus. American Diabetes Association: Clinical Practice Recommendations Committee Report. Diabetes Care 2000;2000:S4-S19.

[30] Matthews DR, Hosker JP, Rudenski AS, Naylor BA, Treacher DF, Turner RC Homeostasis model assessment: insulin resistance and beta-cell function from fasting plasma glucose and insulin concentrations in man. Diabetologia $1985 ; 28: 412-419$.

[31] Taylor KJ, Riely CA, Hammers L, et al. Quantitative US attenuation in normal liver and in patients with diffuse liver disease: importance of fat. Radiology 1986;160:65-71.

[32] Scheuer PJ. Classification of chronic viral hepatitis: a need for reassessment. J Hepatol 1991;13:372-374.

[33] Germani G, Hytiroglou P, Fotiadu A, Burroughs AK, Dhillon AP. Assessment of fibrosis and cirrhosis in liver biopsies: an update. Semin Liver Dis 2011;31:82-90.

[34] Jacobs JE, Brinbaum BA, Shapiro MA, et al. Diagnostic criteria for fatty infiltration of the liver on contrast-enhanced helical CT. AJR 1998;171:659-664.

[35] Fishbein M, Castro F, Cheruku S, et al. Hepatic MRI for fat quantification. J Clin Gastroenterol 2005;39:619-625. 